\title{
Reply to Comment on: Mackin P, Watkinson HM, Young AH (2005) Prevalence of obesity, glucose homeostasis disorders and metabolic syndrome in psychiatric patients taking typical or atypical antipsychotic drugs: a cross-sectional study. Diabetologia 48:215-221
}

Received: 24 March 2005 / Accepted: 30 March 2005 / Published online: 26 May 2005

(C) Springer-Verlag 2005

To the Editor: We thank Dr Engl and colleagues for their comments regarding our recent article [1]. Dr Engl et al are correct to state that there are several other prospective studies that have reported data on glucose homeostasis and weight gain that we did not reference in our article. We do not believe, however, that omitting these references affected the context or conclusions of our study. In our study, which was cross-sectional in design, we compared the prevalence of metabolic disease in patients receiving typical and atypical drugs. The most important finding of our study was the high prevalence of undiagnosed metabolic disease in patients with severe mental illness. Although we did report a higher prevalence of glucose homeostasis disorders and metabolic syndrome in patients taking atypical agents, this difference was not statistically significant. We identified differences with regard to BMI, $H b A_{1} c$ and serum triglycerides between several of the atypical agents, but we were careful to point out that the design of our study did not allow for the direction of causality to be established. Moreover, our study was not designed to elucidate the mechanisms of metabolic dysfunction, and as such, detailed discussion of studies, prospective or otherwise, that have attempted to investigate the pathogenesis of weight gain and glucose intolerance in patients taking antipsychotic drugs would have been otiose.

Dr Engl et al are correct to state that the recent Consensus Development Conference on Antipsychotic Drugs and Obesity and Diabetes [2] (to which we referred in our article) reported that olanzapine and clozapine appear to

P. Mackin $(\bowtie) \cdot$ A. H. Young

School of Neurology, Neurobiology and Psychiatry, University of Newcastle upon Tyne,

Leazes Wing (Psychiatry), Royal Victoria Infirmary,

Newcastle upon Tyne, NE1 4LP, UK

e-mail: Paul.mackin@ncl.ac.uk

Tel.: +44-191-2824382

Fax: +44-191-2820485 be associated with the greatest weight gain. However, the consensus statement also highlights the problem of 'methodology, selection or ascertainment bias, and absence of appropriate or well-characterised control subjects' as well as the fact that many studies are 'limited by relatively short periods of study, by failure to control for a possible treatment sequence bias in "switchover" studies, and by not always using clinically equivalent doses of the medication'. The point is also made that trials of atypical antipsychotics should preferably recruit drug-naïve subjects, and 'study subjects should be well characterised in terms of their baseline risk factors for diabetes, obesity and lipid disorders and their degree of baseline impairment in insulin sensitivity and beta cell function'. Although Dr Engl et al refer to work from their own group, which reported prospective data regarding glucose metabolism in schizophrenic patients receiving olanzapine [3], these data should be considered preliminary given the open-label design of the study, the low number of subjects (ten patients, ten control subjects), the lack of data regarding previous psychotropic medication, and the failure to control for confounding factors (such as physical activity and baseline risk factors for diabetes, etc.).

Metabolic disease in patients with severe mental illness is a major public health issue and the importance of diagnosis and appropriate treatment cannot be overstated. The question of whether severe mental illness is an independent risk factor for metabolic disease remains unanswered. Although differences between the individual atypical agents appear to be emerging with respect to metabolic dysregulation, in the absence of methodologically robust studies of weight gain, glucose homeostasis disorders and dyslipidaemia, it is difficult to draw firm conclusions about the differential effects of these drugs. Well-controlled, prospective, long-term, head-to-head trials of these drugs are urgently needed. Furthermore, such trials should not be restricted to patients with schizophrenia, as antipsychotic drugs are commonly prescribed to patients with other severe mental illnesses such as bipolar disorders. 


\section{References}

1. Mackin P, Watkinson HM, Young AH (2005) Prevalence of obesity, glucose homeostasis disorders and metabolic syndrome in psychiatric patients taking typical or atypical antipsychotic drugs: a cross-sectional study. Diabetologia 48:215-221

2. American Diabetes Association, American Psychiatric Association, American Association of Clinical Endocrinologists, North American Association for the Study of Obesity (2004) Consensus development conference on antipsychotic drugs and obesity and diabetes. Diabetes Care 27:596-601

3. Ebenbichler CF, Laimer M, Eder U et al (2003) Olanzapine induces insulin resistance: results from a prospective study. J Clin Psychiatry 64:1436-1439 